\title{
CONVERGENCE OF INFINITE EXPONENTIALS WITH COMPLEX ELEMENTS
}

\author{
W. J. THRON
}

Let $\left\{t_{n}(z)\right\}$ be a sequence of exponential functions

$$
t_{n}(z)=e^{a_{n} z}
$$

where the $a_{n}$ are arbitrary complex numbers not on the negative real axis. Next define inductively

$$
T_{k, m}(z)=t_{k}\left(T_{k+1, m}(z)\right), \quad T_{m+1, m}(z)=z, \quad 1 \leqq k \leqq m+1, m \geqq 1,
$$

and set

$$
T_{n}(z)=T_{1, n}(z) .
$$

The sequence $\left\{T_{n}(1)\right\}$ will then be called an infinite exponential. An infinite exponential is frequently written as

$$
b_{1}^{b_{3}}{ }^{b_{2}}
$$

where $a_{n}=\log b_{n}$. (Wherever the logarithm is used in this article it is understood to be the principal branch of the function.) However, this way of writing an infinite exponential is ambiguous since it is not clear what value of $z$ is to be used, and since different choices of $z$ may lead to different convergence behavior of the sequence. Instead of $z=1$, we could have used $z=0$ without affecting the convergence behavior of a given infinite exponential since $T_{n}(0)=T_{n-1}(1)$.

The purpose of this article is to prove the following theorem.

THEOREM. An infinite exponential converges if for all $n \geqq 1$

$$
\left|a_{n}\right| \leqq e^{-1}
$$

The value $u$ to which the infinite exponential converges satisfies the condition

$$
|\log u| \leqq 1 .
$$

Received by the editors March 25, 1957. 
For real $a_{n}$ this result is due to Barrow [1], who also coined the term "infinite exponential." For all $b_{n}=b$ the problem goes back to Seidel $[3]$ and Eisenstein.

In the proof we employ a modification of a method developed by the author and others to obtain convergence region criteria for continued fractions. Let $U$ be the region consisting of all $u$ satisfying the condition

$$
|\log u| \leqq 1
$$

Now introduce

$$
A=\bigcap_{u \in U} \frac{\log U}{u} .
$$

(By $f(U)$ we mean the set of all complex numbers $f(u), u \in U$.) The set $A$ then consists of those and only those elements $a$ for which, for any arbitrary element $u_{2}$ of $U$, there exists another element $u_{1} \in U$ such that

$$
a=\frac{\log u_{1}}{u_{2}}
$$

This definition of $A$ insures that

$$
t_{n}(U) \subset U, \quad \text { if } a_{n} \in A .
$$

It is easily verified that, for the given set $U$, the set $A$ consists of those and only those elements $a$ for which

$$
|a| \leqq e^{-1} \text {. }
$$

We now assume that for all $n \geqq 1,\left|a_{n}\right| \leqq e^{-1}$. Let $C$ be the boundary of $U$ and

$$
U_{k, m}=T_{k, m}(U), \quad C_{k, m}=T_{k, m}(C) .
$$

We easily see that $z \in C$ if and only if $z=r e^{i \theta}$, where

$$
r=e^{ \pm\left(1-\theta^{2}\right)^{1 / 2}}, \quad|\theta| \leqq 1 .
$$

The facts that $t_{n}(U) \subset U$ and $1 \in U$ then help to establish the following relations:

$$
\begin{aligned}
U_{k, m+1} & \subset U_{k, m} \subset U, \\
T_{n}(1) & \in U_{1, n} \subset U_{1, m},
\end{aligned}
$$$$
n \geqq m \text {. }
$$

Since the sets $C_{k, m}$ are all simple, closed, and rectifiable curves we can introduce $l\left(C_{k, m}\right)$, the length of $C_{k, m}$. It is then clear that the diameter 
of $U_{k, m}$ is less than $l\left(C_{k, m}\right)$ and therefore

$$
\left|T_{n}(1)-T_{n+p}(1)\right|<l\left(C_{1, m}\right) \quad \text { for } n \geqq m \text { and } p \geqq 0 .
$$

Hence the sequence $\left\{T_{n}(1)\right\}$ satisfies the Cauchy criterion and thus converges, provided $\lim _{m \rightarrow \infty} l\left(C_{1, m}\right)=0$. The value to which it converges must be in $U$ since $T_{n}(1) \in U$, for all $n \geqq 1$, and $U$ is closed. To show that $\lim l\left(C_{1, m}\right)=0$ we make use of the well known formula (see for example [3, p. 155])

$$
l\left(C_{1, m}\right)=\int_{C}\left|T_{m}^{\prime}(z)\right||d z| .
$$

We now split $C$ into two arcs. Let $C^{\prime}(m)$ be that part of $C$ for which $|z| \leqq e\left(1-m^{-1 / 2}\right)$, and let $C^{\prime \prime}(m)$ be the remainder of $C$. We then have

$$
l\left(C_{1, m}\right)=\int_{C^{\prime}(m)}\left|T_{m}^{\prime}(z)\right||d z|+\int_{C^{\prime \prime}(m)}\left|T_{m}^{\prime}(z)\right||d z| .
$$

Now $t_{m}^{\prime}(z)=a_{m} e^{a_{m} z}$, so that for all $a_{m} \in A, z \in U\left|t_{m}^{\prime}(z)\right| \leqq 1$. It follows that

$$
\int_{C^{\prime \prime}(m)}\left|T_{m}^{\prime}(z)\right||d z| \leqq l\left(C^{\prime \prime}(m)\right)
$$

and thus tends to zero as $m$ tends to infinity. To approximate the first integral we observe that for $z \in C_{k, m}^{\prime}$, where $C_{k, m}^{\prime}=T_{k, m}\left(C^{\prime}(m)\right)$, we have

$$
|z| \leqq e r_{k} \leqq e \frac{m^{1 / 2}+m-k}{m^{1 / 2}+m-k+1}, \quad k=1, \cdots, m+1 .
$$

To establish this we note that

$$
e r_{k} \leqq e^{(1 / e)\left(e r_{k+1}\right)}, \quad 1 \leqq k \leqq m,
$$

and hence

$$
r_{k} \leqq e^{r_{k+1}-1} .
$$

Now $r_{m+1}=1-m^{-1 / 2}$, so the formula holds for this value of $k$. To prove it for the remaining $k$, we recall that for all $n>0(1+1 / n)^{n}<e$ and hence $e^{-1}<(1+1 / n)^{-n}$, and proceed as follows:

$$
\begin{aligned}
r_{k} \leqq e^{\tau_{k+1}-1} & \leqq e^{-1 /\left(m^{1 / 2}+m-k\right)} \\
& <\left[(1+1 /(m+m-k))^{-\left(m^{1 / 2}+m-k\right)}\right]^{1 /\left(m^{1 / 2}+m-k\right)} \\
& =\frac{m^{1 / 2}+m-k}{m^{1 / 2}+m-k+1} .
\end{aligned}
$$


Now

$$
\begin{aligned}
\int_{C^{\prime}(m)}\left|T_{m}^{\prime}(z)\right||d z| & =\int_{C^{\prime}(m)} \prod_{k=1}^{m}\left|t_{k}^{\prime}\left(T_{k+1, m}(z)\right)\right||d z| \\
& \leqq l\left(C^{\prime}(m)\right) \prod_{k=1}^{m} \max _{z \in C^{\prime} k+1, m}\left|t_{k}^{\prime}(z)\right| \\
& \leqq l\left(C^{\prime}(m)\right) \prod_{k=1}^{m}(1 / e) e^{\left(m^{1 / 2}+m-k-1\right) /\left(m^{1 / 2}+m-k\right)} \\
& =l\left(C^{\prime}(m)\right) \prod_{k=1}^{m} e^{-1 /\left(m^{1 / 2}+m-k\right)} \\
& \leqq l\left(C^{\prime}(m)\right) \prod_{k=1}^{m}\left(\frac{m^{1 / 2}+m-k}{m^{1 / 2}+m-k+1}\right) \\
& =l\left(C^{\prime}(m)\right) m^{1 / 2} /\left(m^{1 / 2}+m\right) \\
& <l(C) m^{-1 / 2} .
\end{aligned}
$$

This shows that this integral, and hence $l\left(C_{1, m}\right)$, tends to zero as $m$ tends to infinity. The proof of the theorem is now complete. We conclude by observing that our proof could have been greatly simplified if we had been content with proving that the infinite exponential converges for $\left|a_{n}\right| \leqq e^{-1}(1-\epsilon)$, where $\epsilon>0$ can be chosen arbitrarily small.

\section{REFERENCES}

1. D. F. Barrow, Infinite exponentials, Amer. Math. Monthly vol. 43 (1936) pp. $150-160$.

2. Z. Nehari, Conformal mapping, New York, 1952.

3. L. Seidel, Über die Grenzwerte eines unendlichen Potenzausdruckes, Abh. Kgl. Bayr. Akad. 2. Kl. vol. 11 (1870).

UNIVERSITY OF Colorado 